\title{
ESTUDO DO EFEITO DOS ÂNIONS NA DEGRADAÇÃO DO CORANTE REMAZOL VERMELHO RB 133\% POR $\mathrm{H}_{2} \mathrm{O}_{2} / \mathrm{UV}$
}

\author{
STUDY OF THE ANIONS EFFECT OF THE \\ DEGRADATION OF THE DYE REMAZOL RED RB 133\% \\ BY $\mathrm{H}_{2} \mathrm{O}_{2} / \mathrm{UV}$
}

\section{Jefferson Pereira Ribeiro', Juliene Tomé Oliveira², Eliezer Fares Abdala Neto², Francisco Wagner Sousa ${ }^{3}$, Ronaldo Ferreira Nascimento ${ }^{1}$}

${ }^{1}$ Programa de Pós-graduação em Engenharia Civil - Departamento de Engenharia Hidráulica e Ambiental (DEHA-UFC), Fortaleza-Ce, Brasil. jeffersonufc7@gmail.com

${ }^{2}$ Laboratório de Análises de Traços- Departamento de Química Analítica e FísicoQuímica (UFC). LAT-UFC@ yahoogrupos.com.br

${ }^{3}$ Instituto Federal do Ceará Departamento de Química do IFCE-Campus Crateús.

fr_wagner80@yahoo.com.br

\begin{abstract}
RESUMO
As atividades industriais que consomem excesso de água no seu processo industrial, normalmente geram um elevado volume de efluentes, sendo a indústria têxtil um exemplo típico. A oxidação química é um dos processos alternativos para o tratamento de efluentes contendo corantes têxteis, entre eles destacam-se os processos oxidativos avançados (POAs) que são baseados na geração de radicais hidroxilas $(\mathrm{OH})$ no qual são altamente oxidantes, podendo decompor compostos de maneira rápida e não-seletiva, conduzindo a mineralização parcial ou completa do contaminante. Neste trabalho estudou-se o efeito dos ânions na degradação do corante remazol vermelho RB $133 \%$ pelo tratamento combinado $\mathrm{H}_{2} \mathrm{O}_{2} / \mathrm{UV}$. Os resultados das eficiências de remoção de cor ao final do processo indicaram que não houve diferença entre os tratamentos na presença dos ânions na concentração estudada $(10 \mathrm{mM})$, quando comparada a degradação sem a presença desses ânions, pois ao final de todos os tratamentos a solução ficou incolor. Para o parâmetro de demanda química de oxigênio (DQO) houve influência nas eficiências de remoção dos ânions estudados, principalmente para $\mathrm{NO}_{3}{ }^{-}, \mathrm{Cl}^{-}$e $\mathrm{PO}_{4}{ }^{3-}$.
\end{abstract}

Palavras-chave: POA. Descoloração. Corante. Remazol Vermelho RB 133\%. DQO.

\footnotetext{
ABSTRACT

Industrial activities that consume excess water in its manufacturing process, typically generate a high volume of effluents, the textile industry being a typical example. The chemical oxidation is an alternative for the treatment of effluents containing textile dyes, among them stand out from the
} 
advanced oxidation processes (AOPs) are based on the generation of hydroxyl radicals (. OH) in which are highly oxidizing processes and can decompose compounds quickly and non-selective manner, leading to partial or complete mineralization of the contaminant. We studied the effect of anions on the degradation of the dye Remazol Red RB $133 \%$ by combined treatment $\mathrm{H}_{2} \mathrm{O}_{2} / \mathrm{UV}$. The results of the removal efficiencies of color at the end of the process indicated that there was no difference between treatments in the presence of anions in the studied concentration $(10 \mathrm{mM})$ compared the degradation without the presence of these anions, because at the end of all treatments the solution was colorless. For the parameter of chemical oxygen demand (COD) was no influence on the removal efficiencies of the studied anions, especially for $\mathrm{NO}_{3}{ }^{-}, \mathrm{Cl}^{-}$, and $\mathrm{PO}_{4}{ }^{3-}$.

Keywords: POA. Discoloration. Dye. Remazol Red RB 133\%. COD.

\section{INTRODUÇÃO}

A contaminação de águas naturais tem sido apontada como um dos maiores problemas da sociedade moderna. Dentro deste contexto, o setor têxtil apresenta especial destaque, pois associado ao baixo aproveitamento dos insumos (corantes, detergentes, engomantes e amaciantes) gera grandes volumes de efluentes têxteis, devido ao uso excessivo de água (KUNZ et al., 2002).

Os efluentes têxteis caracterizam-se por ser altamente coloridos, devido à presença de corantes que não se fixam na fibra durante o processo de tingimento, além disso, possuem uma grande quantidade de sólidos suspensos, $\mathrm{pH}$ altamente flutuante, temperatura elevada, grandes concentrações de Demanda Química de Oxigênio (DQO) e considerável quantidade de metais pesados (Cr, Ni e Cu) (CISNEROS, et al., 2002). O lançamento destes efluentes no ecossistema aquático pode diminuir a transparência da água e a penetração da radiação solar (HERRMANN et al., 2001).

Existem hoje diversos processos para o tratamento de diferentes tipos de corantes da indústria têxtil. Dentre estes processos comumente utilizados destacam-se os tratamentos físico-químico e o biológico, os quais como outros processos tecnológicos de tratamento de efluentes apresentam vantagens e desvantagens. As desvantagens desses processos é que demandam bastante tempo e apenas transferem de fase os corantes, gerando outro passivo ambiental (lodo), podendo ainda originar traços de dioxinas e furanos como subprodutos de oxidação incompleta (SCHRANK, 2000; NOGUEIRA e JARDIM, 1998). Devido a estas limitações o desenvolvimento de tecnologias mais efetivas e limpas vem sendo incentivadas para o tratamento de efluentes oriundos de indústrias têxteis.

Um método alternativo bastante eficaz e versátil utilizado no tratamento de efluentes têxteis são os Processos Oxidativos Avançados (POAs) (ARAÚJO et al., 2006). Os POAs são tecnologias baseadas na geração de radicais hidroxila (agente oxidante) podendo decompor compostos de maneira rápida e não seletiva conduzindo a mineralização parcial ou completa do contaminante. Os principais métodos utilizados são tratamentos combinados, tais como: $\mathrm{Fe} / \mathrm{H}_{2} \mathrm{O}_{2}, \mathrm{O}_{3} / \mathrm{UV}, \quad \mathrm{O}_{3} / \mathrm{H}_{2} \mathrm{O}_{2}, \mathrm{O}_{3} / \mathrm{TiO}_{2}, \mathrm{O}_{3} / \mathrm{UV} / \mathrm{H}_{2} \mathrm{O}_{2}, \mathrm{O}_{3} / \mathrm{Mn}^{+2}, \mathrm{O}_{3} /$ ultrasom, $\mathrm{H}_{2} \mathrm{O}_{2} / \mathrm{UV}$ (ARAÚJO et al., 2006; ALMEIDA et al., 2004; RIBEIRO et al., 2010).

Embora os $\mathrm{POA}_{S}$ apresentem vantagens, principalmente relacionado à oxidação de compostos, alguns ânions podem modificar a eficiência do processo sequestrando os radicais livres ( $\mathrm{HO}^{\circ}, \mathrm{HO}_{2}{ }^{\circ}$ e $\left.\mathrm{O}_{2}{ }^{-}\right)$. As equações 1-10 mostram como o radical hidroxila é desativado na presença de ânions (GALINDO et al., 2001; GAO et al., 2009). 


$$
\begin{gathered}
\mathrm{HO}+\mathrm{HCO}_{3}^{-} \rightarrow \mathrm{OH}^{-}+\mathrm{HCO}_{3}{ }^{-} \\
\mathrm{HO}+\mathrm{CO}_{3}^{2-} \rightarrow \mathrm{OH}^{-}+\mathrm{CO}_{3}^{--} \\
\mathrm{HO}+\mathrm{PO}_{4}^{3-} \rightarrow \mathrm{OH}^{-}+\mathrm{PO}_{4}{ }^{2} . \\
\mathrm{HO}^{-}+\mathrm{Cl}^{-} \rightarrow \mathrm{ClOH}^{-} \\
\mathrm{ClOH}^{-} \rightarrow \mathrm{HO}+\mathrm{Cl}^{-} \\
\mathrm{ClOH}^{-}+\mathrm{H}^{+} \rightarrow \mathrm{Cl}+\mathrm{H}_{2} \mathrm{O} \\
\mathrm{Cl}+\mathrm{Cl}^{-} \rightarrow \mathrm{Cl}_{2}^{-} \\
\mathrm{Cl}_{2}^{-} \rightarrow \mathrm{Cl}^{-}+\mathrm{Cl}^{-} \\
\mathrm{HO}^{-}+\mathrm{NO}_{3}{ }^{-} \rightarrow \mathrm{HO}^{-}+\mathrm{NO}_{3}{ }^{-} \\
\mathrm{SO}_{4}{ }^{2-}+\mathrm{HO} \rightarrow \mathrm{HO}^{-}+\mathrm{SO}_{4}{ }^{-}
\end{gathered}
$$

Neste trabalho estudou-se o efeito dos ânions na degradação do corante remazol vermelho RB $133 \%$ pelo tratamento combinado $\mathrm{H}_{2} \mathrm{O}_{2} / \mathrm{UV}$.

\section{MATERIAIS E MÉTODOS}

\subsection{Reator fotoquímico}

O estudo de degradação do corante foi realizado em um reator fotoquímico $(95 \mathrm{~cm}$ de comprimento e $2,8 \mathrm{~cm}$ de diâmetro interno) constituída por um tubo de quartzo, com fonte de irradiação ultravioleta (UV) obtida por uma lâmpada de baixa pressão de vapor de mercúrio de 30 watts (Figura 1) (PHILLIPS, 2011).

O corante remazol vermelho RB $133 \%$ foi fornecido pela empresa Dystar e foi usado para os ensaios de degradação. Os experimentos foram realizados em uma vazão de $25\left(\mathrm{~mL} \mathrm{~min}^{-1}\right)$ com um volume total de 2 litros da solução contendo o corante. Nesses experimentos foram usados os sais de bicarbonato de sódio, carbonato de sódio, nitrato de sódio, tiossulfato de sódio, cloreto de sódio e fosfato de sódio. A massa do corante utilizada foi de $2 \mathrm{~g}$. A concentração de cada ânion foi de $10,0 \mathrm{mM}$. O tempo de experimento foi de 250 minutos usando o sistema $\mathrm{H}_{2} \mathrm{O}_{2} / \mathrm{UV}$, sendo que em intervalos de 60 minutos foram realizadas as análises físico- químicas.

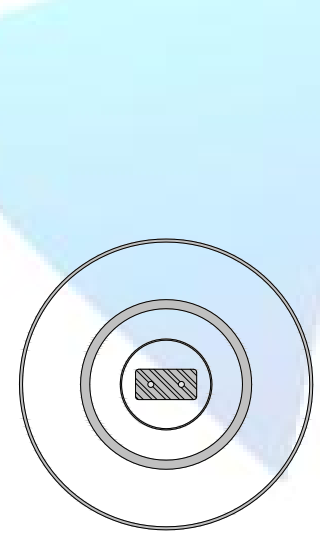

(A)

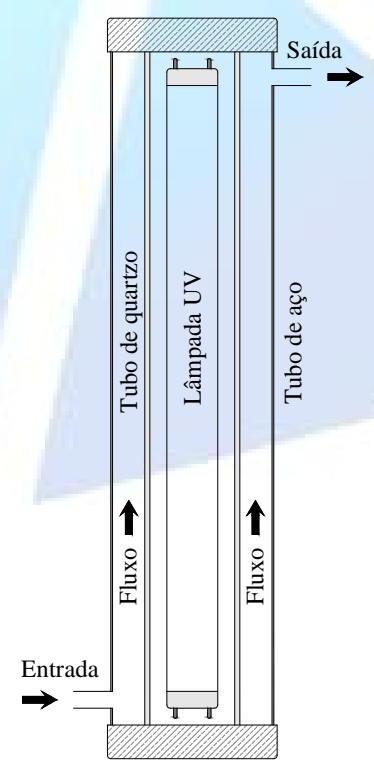

(B)

Figura 1. Esquema Geral do Reator Fotoquímico usado no Tratamento do corante pelo Processo de Oxidação Avançada $\mathrm{H}_{2} \mathrm{O}_{2} / \mathrm{UV}$. Vista Superior (A) e Vista Frontal (B). Fonte: $\mathrm{O}$ autor. 


\subsection{Análises Físico-Químicas}

A caracterização do efluente sintético foi realizado através das análises de cor verdadeira, peróxido residual, $\mathrm{pH}$, condutividade elétrica e DQO. Algumas análises foram baseadas monitorados parâmetros diretamente nos instrumentos de medida, a seguir:

pH TEC-5).

Os valores foram medidos através de pHmetro digital microprocessado TECNAL (modelo

\section{Condutividade} Star).

Os valores foram medidos por um condutivímetro THERMO SCIENTIFIC (modelo Orion 5

\section{Peróxido de hidrogênio $\left(\mathrm{H}_{2} \mathrm{O}_{2}\right)$}

A concentração do peróxido de hidrogênio $\left(\mathrm{H}_{2} \mathrm{O}_{2}\right)$ foi determinada pelo método de titulação volumétrica permanganimetria, conforme Standard Methods (APHA, 2005).

\section{Cor verdadeira}

A cor foi analisada e determinada em um espectrofotômetro (Thermo - Nicolet Evolution 100). Para o corante sintético, foi realizada uma varredura a cada 60 minutos de experimento. As amostras foram previamente diluídas (1:5) em água Milli-Q e, então, eram centrifugadas por 2 minutos a 13000rpm (Eppendorf - Mini Spin). A eficiência de descoloração foi estimada a partir das absorbâncias medidas segundo a Equação 1:

$$
\text { Eficiência }(\%)=\left(1-A_{f} / A_{o}\right) \times 100
$$

Com base nos dados referentes à cor, aplicou-se o modelo de pseudo-primeira ordem utilizando a equação do modelo não- linear (Equação 2):

$$
\left(\mathrm{A}_{\mathrm{f}} / \mathrm{A}_{0}\right)=\mathrm{e}^{-\mathrm{k} . \mathrm{t}}
$$

Onde k é uma constante $\left(\mathrm{min}^{-1}\right)$, t é o tempo de tratamento (minutos) e $\mathrm{A}_{o} e \mathrm{~A}_{f}$ são as absorbâncias iniciais e finais de DQO (mg/L).

\section{Demanda química de oxigênio (DQO)}

A DQO foi determinada fotometricamente (Thermo - Nicolet Evolution 100), pelo método de refluxo fechado (método 5220 D), conforme Standard Methods (APHA, 2005). A concentração residual de peróxido de hidrogênio $\left(\mathrm{H}_{2} \mathrm{O}_{2}\right)$ interfere na medida de DQO ao consumir $\mathrm{K}_{2} \mathrm{Cr}_{2} \mathrm{O}_{7}$ (Equação 3) (TALINLI e ANDERSON, 1992).

$$
\mathrm{Cr}_{2} \mathrm{O}_{7}^{2-}+3 \mathrm{H}_{2} \mathrm{O}_{2}+8 \mathrm{H}^{+} \rightarrow 2 \mathrm{Cr}^{3+}+3 \mathrm{O}_{2}+7 \mathrm{H}_{2} \mathrm{O}
$$

Esta interferência foi corrigida conhecendo-se a DQO de uma solução de peróxido de hidrogênio. Foram realizadas medidas de DQO em soluções de peróxido de hidrogênio de concentração $\left(1 \mathrm{~g} \mathrm{~L}^{-1}\right)$ e, conhecendo-se então a concentração de peróxido de hidrogênio residual, as correções foram realizadas. Lin e Lo (1997) encontraram em seu trabalho que $\left(1 \mathrm{~g} \mathrm{~L}^{-1}\right)$ de peróxido de hidrogênio corresponde a $270\left(\mathrm{mg} \mathrm{L}^{-1}\right)$ de DQO. 


\section{RESULTADOS E DISCUSSÃO}

O estudo dos ânions interferentes vem sendo estudado por diversos pesquisadores (YEBER et al., 2010; YUAN et al., 2011; KALSOOM et al., 2012), pois os mesmos podem retardar o processo de descoloração através do processo de captura dos radicais hidroxila.

Os resultados do estudo de efeito dos ânions tiossulfato $\left(\mathrm{S}_{2} \mathrm{O}_{3}{ }^{2-}\right)$, nitrato $\left(\mathrm{NO}_{3}{ }^{-}\right)$, cloreto $\left(\mathrm{Cl}^{-}\right)$, carbonato $\left(\mathrm{CO}_{3}{ }^{2-}\right)$, bicarbonato $\left(\mathrm{HCO}_{3}{ }^{-}\right)$, fosfato $\left(\mathrm{PO}_{4}{ }^{3-}\right)$ e mistura de ânions podem ser observados pelas Figuras 2 e 3 e Tabela 1. Os resultados das eficiências de remoção de cor ao final do processo (Figura 2) indicaram que não houve diferença entre os tratamentos na presença dos ânions na concentração estudada $(10 \mathrm{mM})$ quando comparada a degradação sem a presença desses ânions, pois ao final de todos os tratamentos a solução ficou incolor. Porém, as taxas de degradação nos mostram que os ânions $\mathrm{S}_{2} \mathrm{O}_{3}{ }^{2-}\left(0,0072 \mathrm{~min}^{-1}\right), \mathrm{NO}_{3}{ }^{-}\left(0,0073 \mathrm{~min}^{-1}\right)$ e mistura de ânions $(0,0068$ $\left.\min ^{-1}\right)$ interferiram negativamente no decorrer do processo de descoloração. Em contrapartida os ânions $\mathrm{Cl}^{-}\left(0,0086 \mathrm{~min}^{-1}\right), \mathrm{CO}_{3}{ }^{2-}\left(0,0094 \mathrm{~min}^{-1}\right), \mathrm{HCO}_{3}^{-}\left(0,0089 \mathrm{~min}^{-1}\right)$ e $\mathrm{PO}_{4}{ }^{3-}\left(0,0081 \mathrm{~min}^{-1}\right)$ aceleraram o processo de descoloração, pois os mesmos apresentaram taxas superiores ao processo de tratamento sem a presença dos ânions $\left(0,0079 \mathrm{~min}^{-1}\right)$. Kalsoom et al. (2012) verificaram comportamento semelhante para os ânions $\mathrm{Cl}^{-}, \mathrm{PO}_{4}{ }^{3-}, \mathrm{CO}_{3}{ }^{2-}$ e $\mathrm{SO}_{4}{ }^{2-}$.

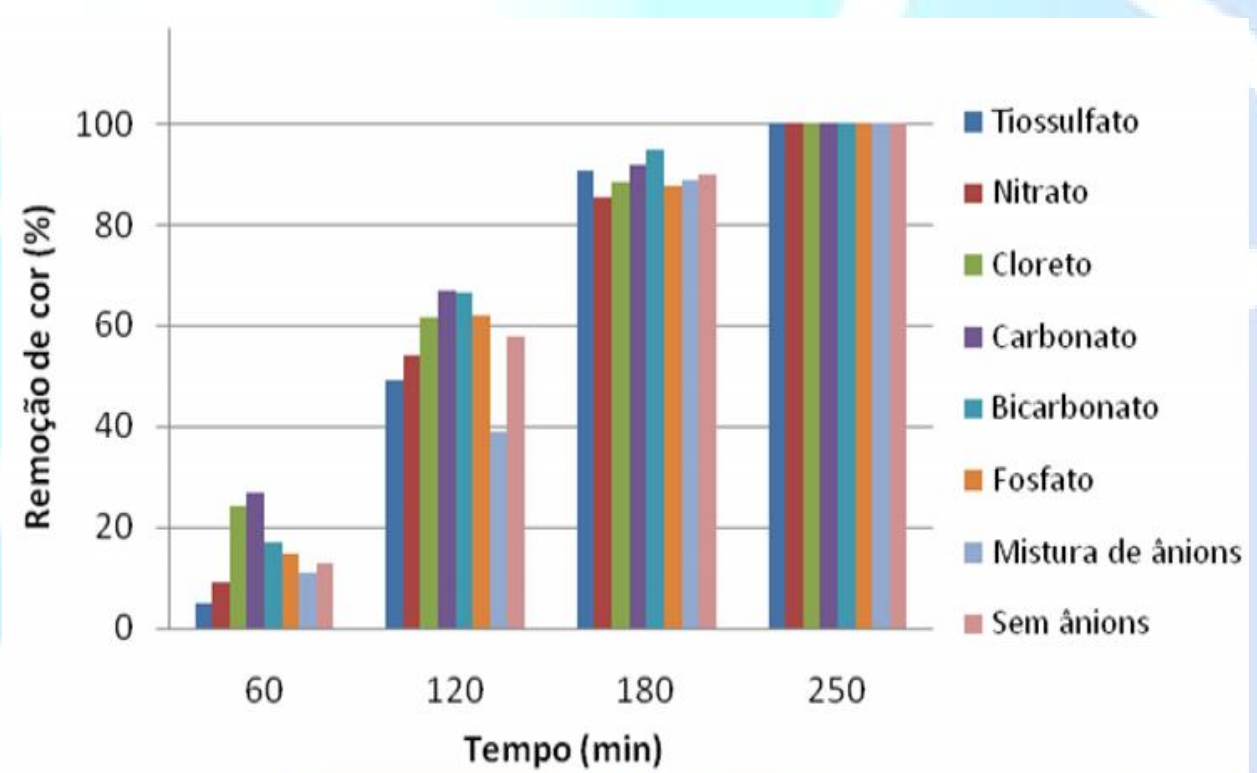

Figura 2. Eficiência de Remoção de Cor Referente ao Estudo do Efeito dos Ânions interferentes. Condições Experimentais: $\mathrm{H}_{2} \mathrm{O}_{2}$ na Linha/UV, pH Inicial 10 e Temperatura de $60{ }^{\circ} \mathrm{C}$.

Os resultados de condutividade mostram um aumento nos valores finais para todos os tratamentos realizados, devido provavelmente à degradação do corante que liberou íons para solução (Tabela 1). Os resultados de peróxido residual mostram um decréscimo nos valores em todos os tratamentos, todavia este efeito é mais notório quando se tem uma maior redução do $\mathrm{pH}$, que foi constatado nos resultados de $\mathrm{NO}_{3}{ }^{-}$e $\mathrm{Cl}^{-}$. Uma das hipóteses para esses baixos valores é a faixa de $\mathrm{pH}$ dos tratamentos que ficaram entre 7-10 com exceção do $\mathrm{S}_{2} \mathrm{O}_{3}{ }^{2-}$, pois a literatura relata que o peróxido de hidrogênio se decompõe mais rapidamente em $\mathrm{pH}$ alcalino (LEGRINI et al., 1993; GALINDO e KALT, 1998). Embora a faixa de pH do tratamento na presença de $\mathrm{S}_{2} \mathrm{O}_{3}{ }^{2-}$ tenha ficado entre 2- 4, o mesmo apresentou um valor final de peróxido próximo ao tratamento na presença de $\mathrm{HCO}_{3}{ }^{-}$. Esse baixo valor nesse tratamento pode está relacionado provavelmente à decomposição do $\mathrm{H}_{2} \mathrm{O}_{2}$ na presença da espécie $\mathrm{S}_{2} \mathrm{O}_{3}{ }^{2-}$ (equação 46 e 47) (YANG et al., 2010; SALARI et al., 2010). 


$$
\begin{gathered}
2 \mathrm{~S}_{2} \mathrm{O}_{3}{ }^{2-}+7 \mathrm{H}_{2} \mathrm{O}_{2} \\
\rightarrow 14 \mathrm{H}^{+}+4 \mathrm{SO}_{4}{ }^{2-}+2 \mathrm{O}_{2} \\
\mathrm{SO}_{4}{ }^{--}+\mathrm{H}_{2} \mathrm{O} \rightarrow \mathrm{SO}_{4}{ }^{2-}+\mathrm{HO}+\mathrm{H}^{+}
\end{gathered}
$$

Tabela 1 - Parâmetros Físico-Químicos monitorados no Estudo com o Tratamento $\mathrm{H}_{2} \mathrm{O}_{2} / \mathrm{UV}$ na Presença de

\begin{tabular}{|c|c|c|c|c|}
\hline Ânions & $\begin{array}{c}\text { Tempo } \\
(\text { min })\end{array}$ & pH & $\begin{array}{c}\text { Condutividade } \\
(\mu \mathrm{S} / \mathrm{cm})\end{array}$ & $\begin{array}{c}\mathrm{H}_{2} \mathrm{O}_{2} \\
\text { residual }(\%)\end{array}$ \\
\hline \multirow{5}{*}{$\mathbf{S}_{2} \mathrm{O}_{3}{ }^{2-}$} & 0 & 10,00 & 2729,50 & 0,00 \\
\hline & 60 & 3,66 & 2815,40 & 0,21 \\
\hline & 120 & 2,32 & 4640,70 & 0,47 \\
\hline & 180 & 2,29 & 5030,20 & 0,61 \\
\hline & 250 & 3,45 & 3060,50 & 0,07 \\
\hline \multirow{5}{*}{$\mathrm{NO}_{3}^{-}$} & 0 & 10,00 & 1388,00 & 0,00 \\
\hline & 60 & 5,94 & 1649,20 & 0,26 \\
\hline & 120 & 4,16 & 1809,80 & 0,41 \\
\hline & 180 & 2,90 & 1945,10 & 0,55 \\
\hline & 250 & 3,36 & 1923,20 & 0,36 \\
\hline \multirow{5}{*}{$\mathrm{Cl}^{-}$} & 0 & 10,00 & 1571,90 & 0,00 \\
\hline & 60 & 6,11 & 1585,30 & 0,39 \\
\hline & 120 & 3,27 & 1648,40 & 0,50 \\
\hline & 180 & 2,95 & 1755,30 & 0,77 \\
\hline & 250 & 3,25 & 1754,70 & 0,51 \\
\hline \multirow{5}{*}{$\mathrm{CO}_{3}{ }^{2-}$} & 0 & 10,00 & 1924,50 & 0,00 \\
\hline & 60 & 9,01 & 2176,30 & 0,28 \\
\hline & 120 & 8,01 & 2283,60 & 0,62 \\
\hline & 180 & 8,29 & 2248,40 & 0,78 \\
\hline & 250 & 7,25 & 2331,80 & 0,13 \\
\hline \multirow{5}{*}{$\mathrm{HCO}_{3}^{-}$} & 0 & 10,00 & 1554,20 & 0,00 \\
\hline & 60 & 9,04 & 1632,60 & 0,26 \\
\hline & 120 & 7,42 & 1620,40 & 0,44 \\
\hline & 180 & 7,60 & 1682,50 & 0,40 \\
\hline & 250 & 7,50 & 1707,10 & 0,04 \\
\hline \multirow{5}{*}{$\mathrm{PO}_{4}{ }^{3-}$} & 0 & 10,00 & 1593,40 & 0,00 \\
\hline & 60 & 7,95 & 1702,70 & 0,33 \\
\hline & 120 & 7,31 & 1716,20 & 0,53 \\
\hline & 180 & 6,79 & 1738,60 & 0,47 \\
\hline & 250 & 7,35 & 1771,20 & 0,17 \\
\hline \multirow{5}{*}{ Mistura de ânions } & 0 & 10,00 & 7223,40 & 0,00 \\
\hline & 60 & 7,80 & 7332,50 & 0,17 \\
\hline & 120 & 7,20 & 7456,70 & 0,34 \\
\hline & 180 & 7,24 & 7475,40 & 0,68 \\
\hline & 250 & 7,43 & 7493,80 & 0,09 \\
\hline
\end{tabular}
Ânions. Condições Experimentais: $\mathrm{H}_{2} \mathrm{O}_{2}$ na linha /UV, pH inicial 10 e temperatura de $60{ }^{\circ} \mathrm{C}$. 
Os resultados da Figura 3 mostram que houve uma redução na taxa de remoção de DQO para os ânions estudados, com exceção do $\mathrm{HCO}_{3}{ }^{-}$. Essa redução na eficiência do processo pode está relacionada provavelmente com a faixa de $\mathrm{pH}$ do tratamento e com a captura dos radicais responsáveis pela oxidação das moléculas.

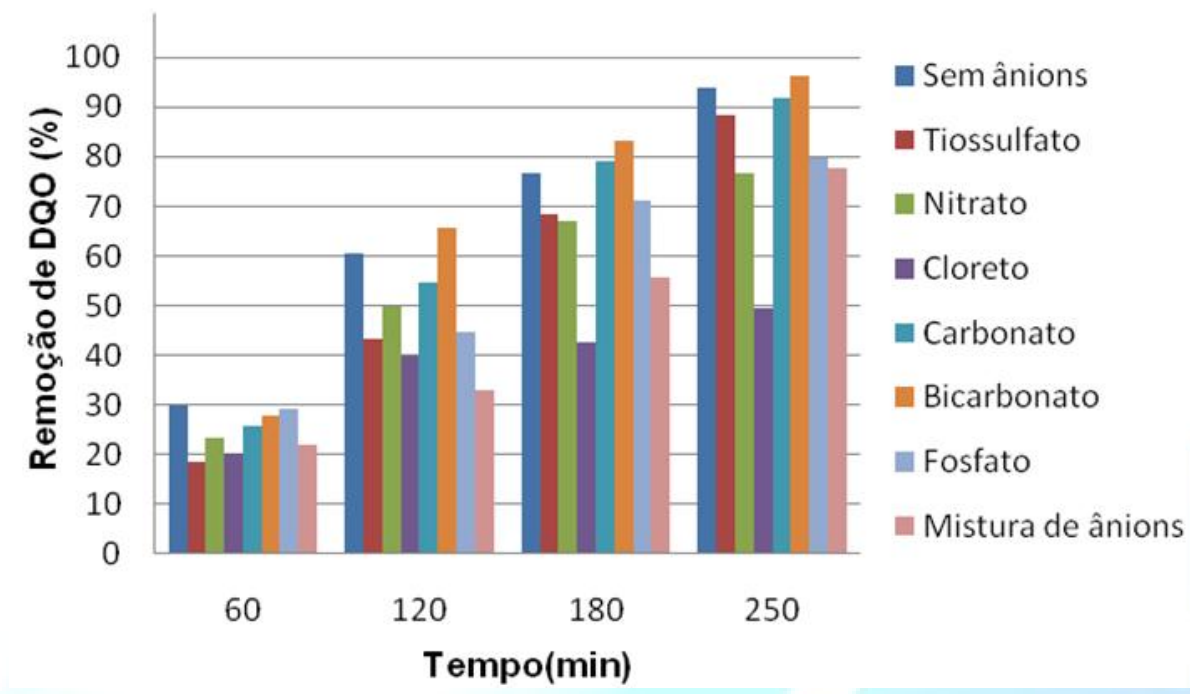

Figura 3. Eficiência de Remoção de DQO Referente ao Estudo do Efeito dos Ânions. Condições Experimentais: $\mathrm{H}_{2} \mathrm{O}_{2}$ na linha/UV, $\mathrm{pH}$ Inicial 10 e temperatura de $60^{\circ} \mathrm{C}$.

É sabido que o pH da solução afeta a distribuição das espécies existentes na solução (OLIVEIRA, 2009). As Figuras 4 - 7 mostram a distribuição das espécies nitrato, cloreto, carbonato, bicarbonato e fosfato de acordo com o valor de $\mathrm{pH}$.

Para o experimento usando o nitrato, o pH variou de um valor inicial de 10 até 2,90. De acordo com a Figura 4, na faixa de $\mathrm{pH}$ em estudo, a espécie $\mathrm{NO}_{3}{ }^{-}$apresenta-se disponível na solução, sendo provavelmente a espécie inibitória no tratamento do corante.

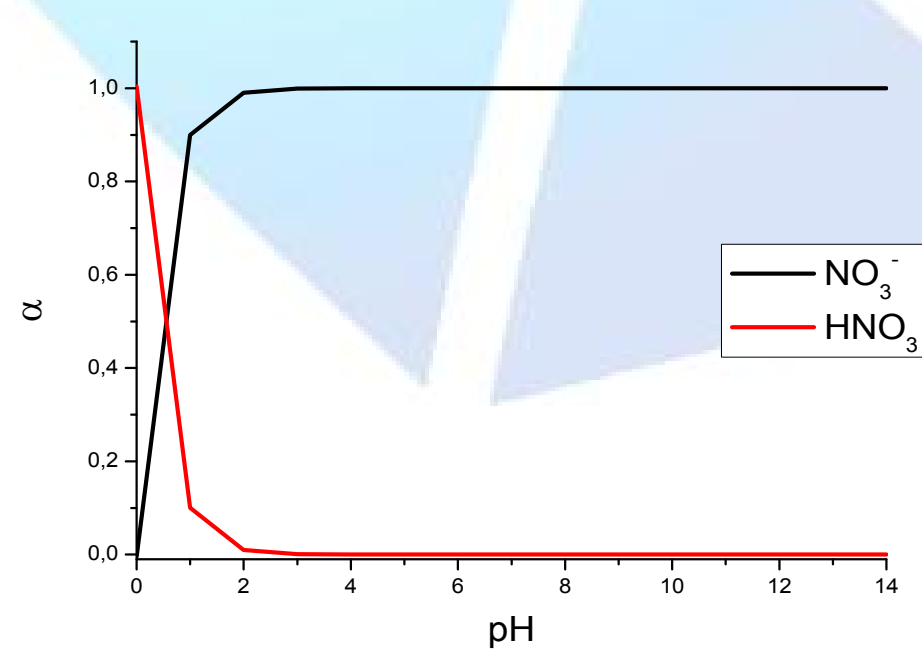

Figura 4. Curva de Distribuição de Espécies do Nitrato. Fonte: Programa Curtipot. 
Para o ânion cloreto, em toda faixa de $\mathrm{pH}$, este apresenta-se disponível na solução (Figura 5), constatando que a espécie química que reduziu a eficiência de remoção de DQO foi o cloreto.

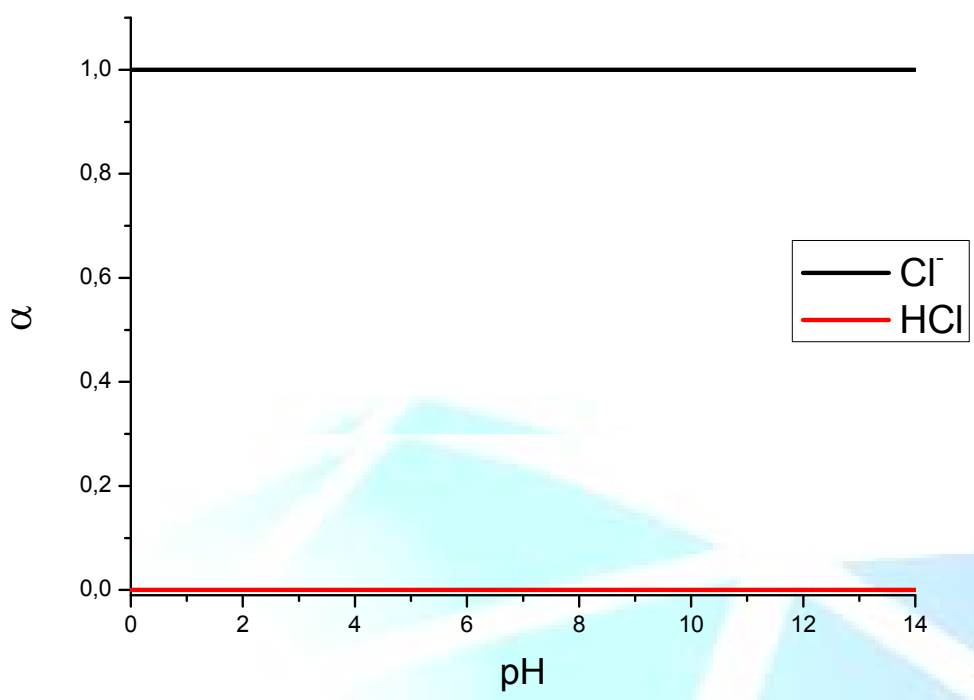

Figura 5. Curva de Distribuição de Espécies do Cloreto. Fonte: Programa Curtipot.

A Figura 6 mostra a distribuição de espécies em função do $\mathrm{pH}$ para os ânions $\mathrm{CO}_{3}{ }^{2-} \mathrm{e}$ $\mathrm{HCO}_{3}{ }^{-}$. Quando o experimento é realizado na presença de carbonato, o $\mathrm{pH}$ inicial da solução decai de 10 até um valor final de 7,25. O experimento na presença de bicarbonato mostra uma variação de 10 (inicial) até 7,5 (final). Ao longo do experimento existe uma variação das espécies presentes, onde em uma hora de tratamento as espécies predominantes na solução são os ânions $\mathrm{CO}_{3}{ }^{2-}$ e $\mathrm{HCO}_{3}{ }^{-}$ para ambos experimentos. Ao final dos tratamentos a espécie $\mathrm{CO}_{3}{ }^{2-}$ não existe na solução, ao passo que as espécies $\mathrm{H}_{2} \mathrm{CO}_{3}$ e $\mathrm{HCO}_{3}{ }^{-}$predominam na solução. É válido salientar que a melhor eficiência do processo com $\mathrm{HCO}_{3}{ }^{-}$se dá provavelmente devido ao tamponamento do sistema provocado pelas espécies presentes que deixam o pH numa faixa ótima de produção de radicais hidroxila.

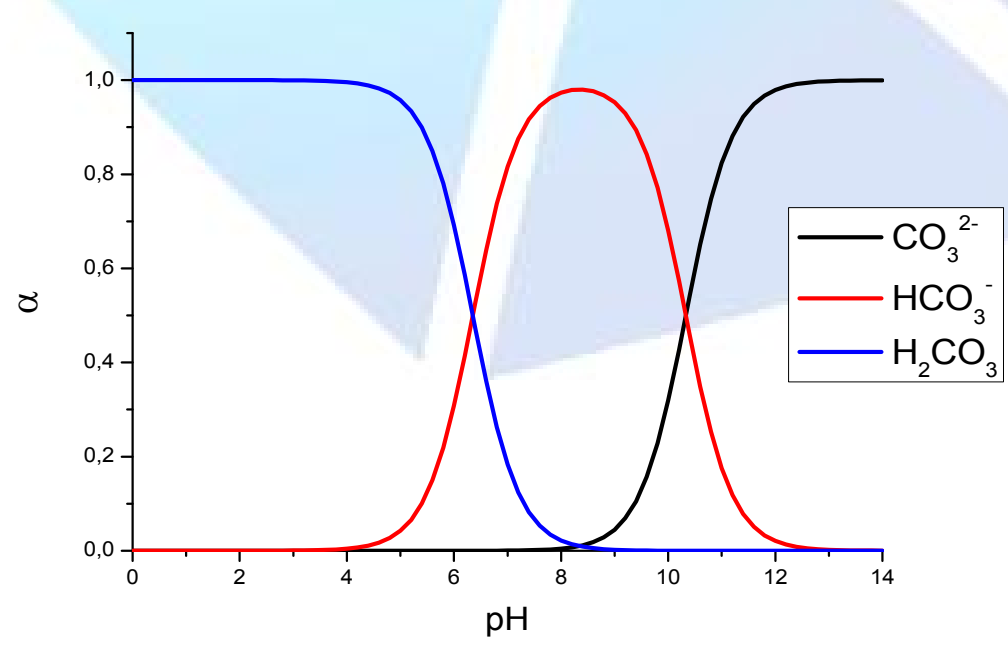

Figura 6. Curva de Distribuição de Espécies do $\mathrm{CO}_{3}{ }^{2-}$ e $\mathrm{HCO}_{3}{ }^{-}$. Fonte: Programa Curtipot. 
No experimento realizado com o fosfato, o pH inicial da solução foi 10 , onde a espécie predominante no meio é $\mathrm{HPO}_{4}{ }^{2-}$ (Figura 7). Ao final do experimento, o pH final é 7,35. Portanto, neste caso, o efeito negativo na eficiência de remoção de DQO não se dá pelo ânion $\mathrm{PO}_{4}{ }^{3-}$, e sim, pelos ânions $\mathrm{H}_{2} \mathrm{PO}_{4}{ }^{-}$e $\mathrm{HPO}_{4}{ }^{2-}$.

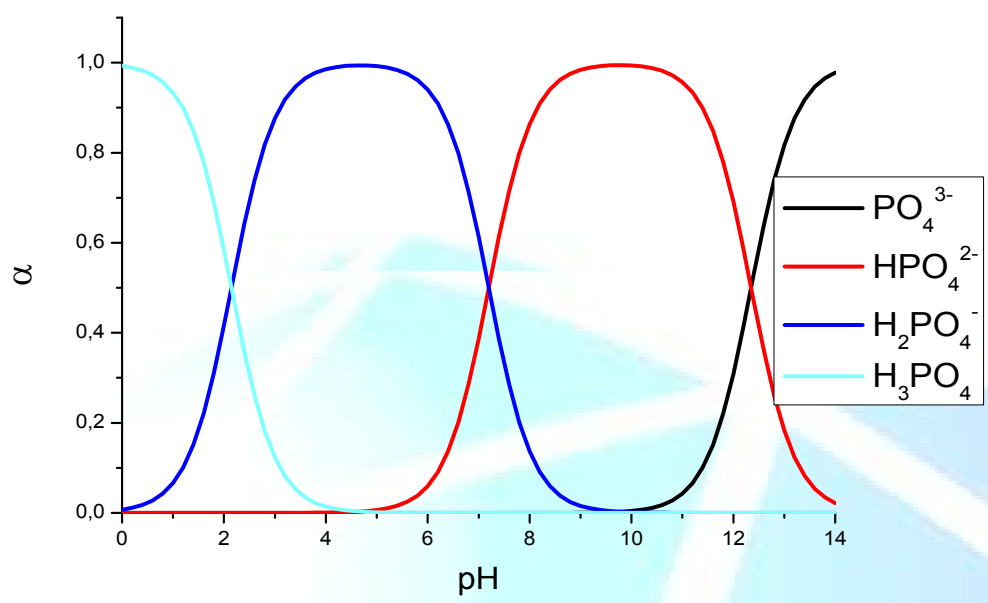

Figura 7. Curva de Distribuição de Espécies do Fosfato. Fonte: Programa Curtipot.

\section{CONCLUSÕES}

Os resultados apresentados mostraram que os ânions pouco influenciaram no processo de descoloração da solução sintética. Em relação à DQO houve uma redução na taxa de remoção para os ânions estudados, com exceção do $\mathrm{HCO}_{3}{ }^{-}$. Os resultados indicam que o processo oxidativo avançado $\mathrm{H}_{2} \mathrm{O}_{2} / \mathrm{UV}$ apresenta grande potencialidade para o tratamento de efluentes contendo corantes têxteis.

\section{AGRADECIMENTOS}

Os autores agradecem ao CNPq e a CAPES pelo suporte financeiro e bolsas concedidas.

\section{REFERÊNCIAS}

ALMEIDA, E.; ASSALIN, M.R.; ROSA, M.A.Tratamento de efluentes industriais por processos oxidativos na presença de ozônio. Química Nova, São Paulo, v. 27, n.5, p.818-824, 2004.

APHA. AWWA, WPCF. Standard Methods for the Examination of Water and wastewater. 21th edition, Washington, USA, 2005.

ARAÚJO, F.V.F.; YOKOYAMA, L. Remoção de cor em soluções de corantes reativos por oxidação com $\mathrm{H}_{2} \mathrm{O}_{2} /$ UV. Química Nova, São Paulo, v. 29, n.1, p.11-14, 2006.

CISNEROS, R.L.; ESPINOZA, A.G.; LITTER, M.I. Photodegradation of an azo dye of the textile industry, Chemosphere, Oxford , v.48, p. 393-399, 2002. 
GALINDO, C.; KALT, A. UV- $\mathrm{H}_{2} \mathrm{O}_{2}$ oxidation of monoazo dyes in aqueous media: a kinetic study. Dyes and Pigments, Vila Real, v.40, p.27-35, 1998.

GALINDO, C.; JACQUES P.; KALT A. Photooxidação of the phenylazonaphtol A020 on $\mathrm{TiO}_{2}$ : kinetic and mechanistic investigations. Chemosphere, Oxford, v.45, p.997-1005, 2001.

GAO, N., DENG, Y., ZHAO, D. Ametryn degradation in the ultraviolet (UV) irradiation/hydrogen peroxide $\left(\mathrm{H}_{2} \mathrm{O}_{2}\right)$ treatment. Journal of Hazardous Materials, Philadelphia PA, v.164, p.640-645, 2009.

GUTZ, I. G. R., Programa Curtipot - pH e Curvas de Titulação Potenciométrica: Análise e Simulação, versão 3.6.1, http://www2.iq.usp.br/docente/gutz/Curtipot.html.

HERRMANN, J.M.; VAUTIER M.; GUILLARD C. Photocatalytic degradation of dyes in water: case study of indigo and of indigo carmine. Journal of Catalysis, Lisboa, v. 201, p.46-59, 2001.

KALSOOM, U.; ASHRAF, S.S.; MEETANI, M.A., RAUF, M.A.; BHATTI, H.N. Degradation and kinetics of $\mathrm{H}_{2} \mathrm{O}_{2}$ assisted photochemical oxidation of Remazol Turquoise Blue. Chemical Engineering Journal, v.200-202, p. 373-379, 2012.

KUNZ, A.; PERALTA-ZAMORA, P.; MORAES, S. G.; DURÁN, N. Novas tendências no tratamento de efluentes têxteis. Química Nova, São Paulo, v. 25, p.78, 2002.

LIN, S.H.; LO, C.C. Fenton process for treatment desizing wastewater. Water Research, v. 31, p. 2050-2056, 1997.

LEGRINI, O.; OLIVEROS, E.; BRAUN, A.M. Photochemical processes for water treatment. Chemical Reviews, v. 93, p. 671-698, 1993.

NOGUEIRA, R.F.P.; JARDIM, W.F. A fotocatálise heterogênea e sua aplicação ambiental. Química Nova, São Paulo, v. 21, p. 69-72, 1998.

OLIVEIRA, A.F. Equilíbrios Ácido-Base em Solução Aquosa: Orientados à Aplicação. $1^{\text {a }}$ Edição, editora Átomo, Campinas, 2009.

PHILIPS. Philips UV purification lamp systems offer highest reliability, enabling maximum protection of water and air quality. Available from:

http://www.lighting.philips.com/pwc_li/main/application_areas/assets/purification/Final_catalogue_ Philips_UV_Purification_April\%202011.pdf, 2011.

RIBEIRO, J.P.; FILHO, N.M.; CORREIA, L.M.; SOUSA, F.W; ARAÚJO, D.S.; NASCIMENTO, R.F. Tratamento combinado POA- Adsorção para remoção de corantes de efluentes aquoso têxtil. Revista DAE, v. 193, p. 4-8, 2010.

SALARI, D.; NIAEI, A.; ABER, A.; RASOULIFARD, M.H. The photooxidative destruction of C.I. Basic Yellow 2 using UV/ $\mathrm{S}_{2} \mathrm{O}_{8}{ }^{2-}$ process in a rectangular continuous photoreactor. Journal of Hazardous Materials, Philadelphia PA, v.166, p.61-66, 2009. 
SCHRANK, S.G. Tratamento anaeróbio de águas residuárias da indústria têxtil. Dissertação (Mestrado em Engenharia Química). Curso de Pós-Graduação em Engenharia Química, UFSC, Florianópolis, 2000.

TALINLI, I.; ANDERSON, G.K. Interference of hydrogen peroxide on the standard COD test. Water Research, v. 26, p.107-110, 1992.

YANG, S.; WANG, P.; YANG, X.; SHAN, L.; ZHANG, W.; SHAO, X.; NIU, R. Degradation efficiencies of azo dye Acid Orange 7 by interaction of heat, UV and anions with commons oxidants: persulfate, peroxymonosulfate and hydrogen peroxide. Journal of Hazardous Materials, Philadelphia PA, v.179, p.552-558, 2010.

YEBER, M. C.; DÍAZ, L.; FERNÁNDEZ, J. Catalytic activity of the SO4 ${ }^{\bullet-}$ radical for photodegradation of the azo dye Cibacron Brilliant Yellow 3 and 3,4-dichlorophenol: Optimization by application of response surface methodology. Journal of Photochemistry and Photobiology A: Chemistry, v. 215, p. 90-95, 2010.

YUAN, R.; RAMJAUN, S. N.; WANG, Z.; LIU, J. Effects of chloride ion on degradation of Acid Orange 7 by sulfate radical-based advanced oxidation process: Implications for formation of chlorinated aromatic compounds. Journal of Hazardous Materials, Philadelphia PA, v.196 p.173179, 2011. 